\title{
FACTORES QUE EXPLICAN LA LEALTAD HACIA UNA EMPRESA DE COMERCIO EN LÍNEA EN MÉXICO, EN TIEMPOS DE CRISIS SANITARIA
}

\author{
FACTORS THAT EXPLAIN LOYALTY TOWARDS AN ONLINE TRADING \\ COMPANY IN MEXICO, IN TIMES OF HEALTH CRISIS
}

\author{
Juan Gabriel Figueroa-Velázquez ${ }^{1} *$ (D); Arlen Cerón-Islas $^{2}$ (D); María Inés Cerón-Montúfar ${ }^{3}$ (D). \\ 1.Universidad Autónoma del Estado de Hidalgo, México.jfigueroa@uaeh.edu.mx \\ 2.Universidad Autónoma del Estado de Hidalgo, México. arlenc@uaeh.edu.mx \\ 3.Universidad Autónoma del Estado de Hidalgo, México. mo243144@uaeh.edu.mx
}

*Autor de Correspondencia: Juan Gabriel Figueroa-Velázquez, correo electrónico:jfigueroa@uaeh.edu.mx

\section{RESUMEN}

Actualmente las empresas están innovando la forma en la que ofrecen sus productos y servicios haciendo uso de la amplia gama de tecnologías existentes, ganando mayor relevancia las plataformas digitales. Es por ello que la lealtad de los clientes, es uno de los elementos de análisis más relevantes dentro de las plataformas digitales por el fácil cambio de preferencia. Por ello esta investigación validó un modelo, mediante la técnica del modelado de ecuaciones estructurales para verificar la influencia que tiene las variables usabilidad de la plataforma, satisfacción y la confianza del cliente sobre la lealtad hacia una plataforma de comercio electrónico en México. La investigación es cuantitativa, de tipo trasversal y explicativa. El análisis de los resultados se llevó a cabo con información proporcionada por 382 usuarios de una plataforma de comercio digital y mediante la obtención de un modelo que cumplió los requisitos de validez convergente y discriminante, y con un nivel de ajuste razonable se encontró que las variables satisfacción y confianza de los consumidores tienen una influencia positiva y significativa con la lealtad de los consumidores de la plataforma, mientras que la variable usabilidad de la plataforma no experimentó significancia estadística.

Palabras clave: lealtad a la empresa; usabilidad; satisfacción; confianza; SEM.

Cómo citar:

Figueroa-Velázquez, Juan Gabriel; Cerón-Islas, Arlen; Cerón-Montúfar, María Inés. (2021). Factores que explican la lealtad hacia una empresa de comercio en línea en méxico, en tiempos de crisis sanitaria. Revista de Investigaciones Universidad del Quindio, 33(S1), 212-224. https://doi.org/10.33975/riuq.vol33nS1.493

\section{Revista de Investigaciones Universidad del Quindío, 33(S1), 212-224; 2021.




\begin{abstract}
Currently, companies are innovating the way in which they offer their products and services making use of the wide range of existing technologies, with digital platforms gaining more relevance. That is why customer loyalty is one of the most relevant analysis elements within digital platforms due to the easy change of preference. For this reason, this research validated a model, using the technique of modeling structural equations to verify the influence that the variables usability of the platform, satisfaction and customer trust have on loyalty towards the electronic commerce platform in Mexico. The research is quantitative, transversal and explanatory. The analysis of the results was carried out with information provided by 350 users of a digital commerce platform and by obtaining a model that met the requirements of convergent and discriminant validity, and with a reasonable level of adjustment, it was found that the variables Consumer satisfaction and trust have a positive and significant influence on the platform's consumer loyalty, while the platform usability variable did not experience statistical significance.
\end{abstract}

Keywords: company loyalty; usability; satisfaction; trust; SEM.

\title{
INTRODUCCIÓN
}

Durante los últimos años el comercio electrónico se ha venido incrementando de manera importante en México, la participación de dicha actividad en el Producto Interno Bruto del país (PIB) pasó de $3.0 \%$ a $5.0 \%$ en 2018, de acuerdo a los datos más recientes del Instituto Nacional de Estadística y Geografía INEGI (2018), ya que hoy en día las empresas están comprando y vendiendo en línea, incluso a nivel internacional por lo que el valor del mercado del comercio electrónico está creciendo, así como el aumento de sus usuarios por la comodidad y variedad de productos que se ofrecen (Organización para la Cooperación y el Desarrollo Económicos, 2018).

Siguiendo la misma línea y de acuerdo a la Asociación de Internet MX (2019) la generación millenial son quienes más realizan compras digitales, entre lo más comprado en internet se encuentra: trasporte con un $65 \%$, comida rápida $47 \%$, alojamiento $43 \%$, viajes $41 \%$, boletos de eventos $40 \%$, descargas digitales $35 \%$, ropa y accesorios $34 \%$ entre otros, los menos adquiridos son software y computadoras $14 \%$, libros $18 \%$ y despensa $23 \%$. En cuanto a la recompra o repetición de esta actividad se encuentra que el $91 \%$ de los compradores volverían a hacerlo. Sin embargo, parte de los usuarios que no volverán a comprar, es debido a la falta de información y transparencia en los comercios donde han comprado (Asociación Mexicana de Internet, 2019).

El comercio electrónico se ha convertido en una nueva modalidad que impacta en las transacciones económicas, que va ganando terreno en todas las esferas de la economía, que tiene como principal característica permitir a los consumidores contar con una gran gama de posibilidades para realizar sus compras, pues al hacer uso de medios electrónicos, pueden acceder a un sinfín de sitios web o plataformas que les permitan obtener mejores beneficios como calidad, precio, promociones de los bienes o servicios que se ofrecen (González, 2020).

Ubaque (2020) plantea que la forma de consumo actual se ha visto influida por los cambios socioculturales y los avances en el pensamiento de requerimientos específicos del consumidor; esto 
debido a que con más frecuencia se tiene acceso a noticias e información disponible en línea, a través de dispositivos o redes sociales que influye directamente en la toma de decisiones de su compra (Peter y Olson, 2006).

En particular, dado el contexto actual dominado por una pandemia a causa del virus SAR COVID-19, Ortega (2020) reporta que existe una relación significativa entre los cambios de hábitos de consumo y los factores del comportamiento del consumidor en tiempos de COVID-19; resaltando que el consumidor ha destinado sus compras en línea, ha desarrollado actividades productivas y busca la satisfacción de sus necesidades; además menciona que las empresas deben diseñar estrategias de marketing digital enfocadas al cliente, generando confianza en el consumidor dado el confinamiento producido.

En este sentido Cuevas (2020), menciona que el COVID-19 ha planteado un nuevo esquema de comprar y vender, ahora existen todo tipo de plataformas digitales que permiten hacer todo tipo de transacciones comerciales, que acercan a compradores y vendedores, generando ahorros de desplazamiento y un rápido conocimiento de oferta y demanda.

\section{Planteamiento del problema}

De acuerdo a la literatura revisada, se han llevado a cabo diversas investigaciones empíricas recientemente que abordan al constructo de la lealtad del consumidor con variables como la satisfacción del consumidor (Lacaci y Martínez, 2017; Phani y Prasanna, 2016; Ramirez, 2015), confianza del cliente (Molinillo et al., 2016; Piscoya, 2019), usabilidad de la plataforma (Celia y Isaza, 2019; Pérez Pallarés, 2018), imagen de marca (Celia y Isaza, 2019; Rodríguez et al, 2017), entre otras, sin embargo, no se encontraron investigaciones que incluyeran de manera conjunta a la usabilidad de la página electrónica de la tienda, la satisfacción y la confianza de los clientes y su relación con la lealtad a la empresa, variables que incorpora la presente investigación. Así mismo, particularmente en México los trabajos referentes a factores que influyen en la lealtad hacia las tiendas en línea son escasos (López et al, 2018). En la misma línea Molinillo, et al., (2016) expresa que aún existen pocos trabajos que relacionen el estado afectivo, la satisfacción y la confianza de los consumidores y su vínculo con la lealtad de las empresas en el contexto del comercio electrónico.

Fuentes et al. (2017) hace hincapié en realizar más investigaciones que permitan identificar aquellas variables más valoradas por los clientes, para poder ser implantadas por los gestores de establecimientos minoristas. En la misma línea, investigadores expresan que resultados obtenidos por investigaciones empíricas, pueden ser incorporada en la planeación de marketing encaminada a aumentar la lealtad del cliente de la tienda virtual (Parra, 2019).

En vista del contexto antes descrito, la presente investigación propone la validación de un modelo, a través de la técnica estadística denominada modelado de ecuaciones estructurales, que explique la influencia de la usabilidad de la página electrónica, la satisfacción y confianza de los consumidores sobre la lealtad de los clientes, tomando como caso de estudio a la empresa de comercio electrónico denominada Mercado Libre en México. 


\section{MARCO TEÓRICO}

\section{Lealtad a la empresa}

La lealtad del consumidor es uno de los elementos de análisis más relevantes dentro de los negocios que tienen presencia en línea, ya que actualmente los consumidores tienden a cambiar fácilmente de plataforma o tienda por aquella que le brinde mejores precios en productos similares (Chou et al., 2015), derivado de ello se recomienda a los gerentes de mercadotecnia planear e implementar estrategias que tenga como objetivo la mejora de las variables que permitan medir y elevar la lealtad de los clientes (López et al, 2018). Este constructo tiene diversas variables que lo determinan, entre las que seencuentran satisfacción del consumidor, confianza a la empresa, seguridad percibida y la usabilidad de la plataforma, por mencionar algunas (Manzano et al., 2010).

Keller (1993; citado por Manzano et al., 2010), sugiere que la lealtad se presenta cuando existen actitudes favorables hacia una marca, que se manifiestan en una actitud de compra repetida y traslado a los establecimientos virtuales como una actitud favorable del consumidor hacia una empresa como un comportamiento repetido de uso y compra (Anderson y Srinivasan, 2003). Por otro lado, Dick y Basu, 1994; Oliver, 1999; Martín y Rodríguez, 2001, (citados en Colmenares y Saavedra, 2007), la definen como la preferencia del usuario por alguna plataforma en particular y menciona que se da mediante 3 fases: primero valora la toma de decisiones que posteriormente se trasforman en actitudes y emociones hacia una plataforma para finalmente convertirse en comportamientos definitivos y recurrentes de compra.

Aslam et al. (2020) investigaron la confianza y la lealtad del cliente digital enfocado en la modalidad B2C, en un estudio realizado a usuarios de tiendas en línea en Pakistán, en el cual se relacionó a la lealtad con las siguientes variables; confianza, calidad de la información, calidad de la interfaz de usuario, privacidad percibida, riesgo de seguridad percibido y conciencia del comercio electrónico. Los resultados arrojaron que existe relación entre las variables antes mencionada, dándole mayor ponderación a la interfaz de los sitios web como el factor que tiene un papel importante en la construcción de la confianza, de manera que una vez que el consumidor comienza a confiar en el sitio web según la calidad de la interfaz tiende a ser más leal a la marca.

\section{Usabilidad de la plataforma}

El verdadero alcance del internet radica en que este permite mayor globalización, por ello es una necesidad además de una cuestión estratégica, la fácil accesibilidad a la plataforma, teniendo en cuenta el poco o mucho conocimiento tecnológico que el usuario tenga. Puede considerarse a la usabilidad como un atributo de la plataforma digital que utiliza la tienda para ofrecer su mercancía, para determinar qué tan fácil es de utilizar la interfaz gráfica, e incluye elementos como la accesibilidad, legibilidad, navegabilidad, facilidad de aprendizaje, velocidad y eficiencia del usuario, es decir la parte heurística de la interfaz de usuario (Sánchez, 2011). En su investigación, Rodríguez et al., (2017) confirman la relación de la usabilidad y satisfacción del cliente como elemento base para el alcance de la lealtad del consumidor, así como enfatizar en la buena valoración de la usabilidad de la plataforma como una variable para medir el índice de lealtad. Así mismo, Pérez (2018) confirmó la relación entre la usabilidad de la plataforma con la satisfacción del cliente, que posteriormente se convierte en confianza y finalmente logra la lealtad del consumidor, además de determinar que los elementos de mayor influencia para los 
consumidores respeto a la usabilidad es la arquitectura de la información y la estética de la plataforma. La investigación realizada por Marín (2017) refuerza que los elementos que deben darle mayor peso en la variable de usabilidad son: la búsqueda de productos concretos ya que al no tener claras las categorías y no tener una imagen de referencia que nos remita al producto en cuestión se necesita de una imagen que ayude a hacer más intuitivo el proceso de compra. Por lo anteriormente expuesto, se puede plantear la siguiente hipótesis:

H1: Existe una influencia entre la usabilidad de la plataforma y la lealtad de los clientes a la tienda en línea.

\section{Confianza del cliente en la plataforma}

En el ámbito de las relaciones entre empresas y consumidores, el principal factor al que se enfrenta el consumidor cuando quiere realizar un intercambio comercial es la falta de información completa sobre los productos y los comportamientos de la empresa, de ello deriva la importancia de la generación de la confianza en el consumidor ya que este suele experimentar los temores asociados con la incertidumbre y miedos ante la posibilidad de la mala práctica (Sanz et al., 2009). La escasa confianza mostrada en por parte de consumidores para realizar sus compras en internet es una de las razones que explica la difícil implantación del comercio electrónico (Hampton y Koufaris, 2005; Wang y Emurian, 2005).

De acuerdo a Pavlou y Gefen (2004; citado por Vega, 2015) la confianza es la situación sobre la que un consumidor tiende a aceptar cierto grado de vulnerabilidad basado en las transacciones con un vendedor. Con frecuencia se asocia la satisfacción del cliente con la confianza ya que ya son las expectativas del consumidor las que definirán su grado de satisfacción al ser comparada con los resultados obtenidos marcado una referencia, así que si dichas expectativas se cumplen el consumidor se siente satisfecho, mejorando su nivel de confianza (Bauer et al., 2002; Sanz et al., 2009).

Sánchez y Montoya (2016) mencionan que la confianza es un aspecto estratégico de la gestión empresarial moderna especialmente en el entorno del comercio electrónico, además, destacan la importancia de la confianza como instrumento favorecedor. En ese estudio se pudo identificar que los factores más influyentes en ella son la reputación del vendedor, riesgo percibido, privacidad de los datos, seguridad en la transacción, asociación y el género del comprador, factores que si se mejoran al momento de entablar una relación con los clientes a través de medios electrónicos, permitirán una mayor tasa de conversión y por ende una mayor utilidad.

Investigaciones empíricas han dado cuenta de la relación entre la confianza de los consumidores con la lealtad a la empresa. Fuentes et al., (2017), en su investigación desarrollada entre los clientes de una tienda minorista en línea en España, encontraron que los consumidores que mostraban confianza en sus compras presentaban mayores posibilidades de ser retenidos y realizar recompras. Es por ello que, de acuerdo a lo previamente comentado, se plantea la siguiente hipótesis:

H2: Existe una influencia positiva y significativa entre la confianza del cliente digital y la lealtad a la tienda en línea.

\section{Satisfacción del cliente}

En la actualidad lograr la plena satisfacción del cliente es un requisito indispensable para lograr 
un buen posicionamiento en la mente de los consumidores, además de ser el primer elemento que antecede a lograr mayores beneficios empresariales como la lealtad del cliente (Thompson, 2006). Kotler y Armstrong (2008) definen a la satisfacción como un estado de ánimo que se adquiere posterior al consumo de un producto o servicio, resultado de la comparativa entre las expectativas y el rendimiento percibido. En cuanto a esta afirmación se mencionan tres posibles resultados; si la comparativa coincide el cliente resultará satisfecho, si la comparativa es baja quedará insatisfecho y por último si la comparación supera sus expectativas quedará encantado (Badía et al., 2018). Lacaci y Martínez (2017) establecen que la relación entre la satisfacción y la lealtad en las plataformas digitales deben enfocarse primeramente en aumentar la satisfacción de los clientes con la finalidad de establecer percepciones positivas, estos al quedar satisfechos presentan mayores posibilidades de volver y seguir comprando en la plataforma o sitio web, manteniendo su lealtad a la empresa. Por su parte, investigaciones empíricas han reportado la relación entre estas dos variables. Un su trabajo Lacaci (2017), encontró una relación positiva entre la satisfacción y la lealtad de los clientes de una plataforma digital minorista en España. En la misma linea, Fuentes et al., (2017) encontraron una relación positiva y significativa entre la satisfacción de los consumidores con una edad media de 41 años, de tiendas minoristas de diferentes tipos de mercancías, con la recomendación boca-oído. Es por ello que, de acuerdo a lo anterior, se puede plantear la siguiente hipótesis:

H3. Existe una relación positiva y significativa entre la satisfacción del cliente y la lealtad a una empresa de comercio digital.

En función a las hipótesis planteadas y a la literatura revisada, se propone el siguiente modelo conceptual:

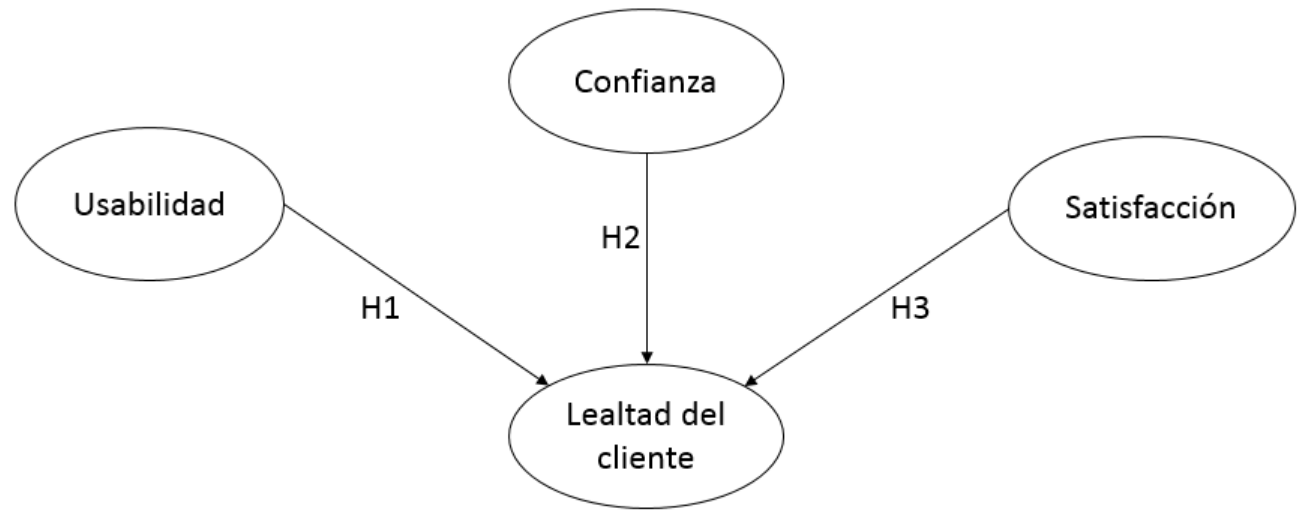

Figura 1. Modelo conceptual propuesto

Fuente: Elaboración propia

\section{METODOLOGÍA}

\section{Diseño de la investigación}

La presente investigación tiene un alcance cuantitativo ya que nos ofrece la posibilidad de generalizar los resultados más ampliamente, nos otorga control sobre los fenómenos y un punto de vista de conteo y magnitudes de éstos (Hernández et al., 2004) referentes a la lealtad a la empresa en plataformas digitales, así mismo es de tipo explicativo, ya que buscó validar la influencia de 
la usabilidad, la confianza y la satisfacción de los clientes sobre la lealtad a una empresa digital. El muestreo es de tipo no probabilístico y de diseño trasversal (Müggenburg y Pérez, 2007). El lugar fijado para la investigación es el Estado de Hidalgo en México, que cuentan con 2,858,359 habitantes. Posteriormente se diseñó un cuestionario online en Google forms, destinado a usuarios de la plataforma Mercado Libre. El cuestionario incluye las escalas de medida de la confianza del cliente en la plataforma, satisfacción del cliente, usabilidad de la plataforma y cuestionamientos acerca de la lealtad a la empresa, las cuales conforman 19 Ítems con una escala Líkert de 5 puntos, además de preguntas relacionadas con las características demográficas de los encuestados. Para esta investigación se estudiaron a usuarios de la plataforma Mercado Libre que radiquen en los Municipios del Estado de Hidalgo, México, por lo que se excluyen a las personas de otros estados aún cuando el estudio se realice en línea. Se encuestaron a 382 usuarios de esta plataforma de comercio en línea, durante los meses de julio a diciembre de 2020, periodo caracterizado por restricciones a la movilidad de las personas y cierres temporales al comercio en tiendas físicas motivadas por la pandemia generada por el virus denominado COVID-19. Para efectos de esta investigación, en la conformación de la muestra no se excluye a los sujetos de estudio por factores como edad, género, escolaridad u otra característica de tipo demográfico. Se utilizaron diferentes escalas validadas para obtener la información necesaria en la investigación. Por lo que para medir la lealtad del consumidor se utilizaron 5 ítems propuestos por Piscoya (2019) en base a los propuestos por Aldás, Lassala, Ruiz y Sanz (2011); para la satisfacción del cliente se midieron con las escalas adaptadas por Fuentes, et al. (2017) y Lacaci (2017); para la confianza del cliente se utilizaron 4 ítems utilizados por Vega (2015) y finalmente para la variable usabilidad de la plataforma se manejaron 4 ítems planteados por Aslam et al. (2020).

Para validar la influencia que generan las variables independientes sobre la lealtad de los clientes a la plataforma electrónica de Mercado Libre en México, se apoyó en la técnica estadística denominada modelado de ecuaciones estructurales, la cual es reconocida como una estadística multivariada que permite confirmar una relación entre dos o más variables, en base a un marco teórico propuesto (Medrano y Muñoz, 2017).

\section{RESULTADOS}

\section{Características de la muestra}

Se obtuvieron un total de 382 encuestas válidas de personas que manifestaron haber realizado al menos una compra en la plataforma estudiada, de las cuales fueron mujeres el $49 \%$, por otra parte el $51 \%$ de la muestra expresó contar con una edad entre 20 y 30 años, así mismo, el 56\% comentó que su gasto en cada compra realizada osciló entre los $\$ 3,001$ y 5,000 pesos mexicanos (Ver tabla 1).

Tabla 1. Características de la muestra

\begin{tabular}{|c|c|c|}
\hline Criterio & Frecuencia & $\%$ \\
\hline \multicolumn{3}{|l|}{ Género } \\
\hline Femenino & 189 & 49 \\
\hline Masculino & 193 & 51 \\
\hline $\mathrm{n}$ & 382 & 100 \\
\hline \multicolumn{3}{|l|}{ Edad } \\
\hline 20 a 30 años & 195 & 51 \\
\hline
\end{tabular}




\begin{tabular}{lcc}
\hline 31 a 40 años & 121 & 32 \\
\hline 41 a 50 años & 42 & 11 \\
\hline Más de 50 años & 24 & 6 \\
\hline $\mathrm{n}$ & 382 & 100 \\
\hline Gasto por compra $(\$)$ & & \\
\hline Menos de $\$ 1,000$ & 48 & 13 \\
\hline De $\$ 1,001$ a $\$ 3,000$ & 93 & 24 \\
\hline De $\$ 3,001$ a $\$ 5,000$ & 215 & 56 \\
\hline Más de $\$ 5,000$ & 26 & 7 \\
\hline $\mathrm{n}$ & 382 & 100 \\
\hline
\end{tabular}

Fuente: Elaboración propia.

\section{EI modelo de medida}

De acuerdo a la literatura revisada, en el modelado de ecuaciones estructurales se identifican dos apartados principales, el modelo de medida y el modelo estructural. El primero representa las relaciones de los constructos con sus respectivos indicadores, mientras que el segundo muestra la interrelación resultante entre los constructos (Cupani, 2012). Para verificar que tanto los indicadores en su conjunto representan a sus respectivos constructos, se verificó la validez convergente y discriminante del modelo de medida. Se utilizó el software Stata en su versión 15 para evaluar la fiabilidad de los constructos mediante el Alfa de Cronbach y la fiabilidad compuesta observándose que los cuatro constructos se encuentran por arriba de 0.7 , el cual se considera como el umbral mínimo comúnmente aceptado (Hair et al., 2010). La literatura revisada sugiere como medida complementaria de la fiabilidad de los constructos, a la varianza media extraída (AVE), la cual se recomienda que deba estar por encima del 0.5 (Hair et al., 2010), con lo cual se observa en la tabla 2, que los tres indicadores cumplen con los niveles mínimos aceptables.

Tabla 2. Indicadores de fiabilidad de los constructos

\begin{tabular}{lccc}
\hline \multicolumn{1}{c}{ Constructo } & Alfa de Cronbach & Fiabilidad compuesta & AVE \\
\hline Satisfacción & 0.92 & 0.93 & 0.68 \\
\hline Usabilidad & 0.85 & 0.86 & 0.61 \\
\hline Confianza en la plataforma & 0.84 & 0.85 & 0.59 \\
\hline Lealtad & 0.88 & 0.88 & 0.60 \\
\hline
\end{tabular}

Fuente: Elaboración propia

Por otra parte, para verificar la validez discriminante se recurrió a la prueba Fornell y Larcker (1981), la cual establece que la AVE debe ser mayor que el cuadrado de las correlaciones entre constructos, prueba que cubre satisfactoriamente el modelo de medida propuesto, tal como se observa en la tabla 3.

Tabla 3. Prueba de validez discriminante del modelo

\begin{tabular}{lccccc}
\hline & Constructo & $\mathbf{1}$ & $\mathbf{2}$ & $\mathbf{3}$ & $\mathbf{4}$ \\
\hline 1 Satisfacción & 0.68 & & & \\
\hline 2 Usabilidad & 0.62 & 0.61 & & \\
\hline 3 Confianza & 0.64 & 0.52 & 0.59 \\
\hline
\end{tabular}




\begin{tabular}{lllll}
\hline 4 Lealtad & 0.61 & 0.46 & 0.56 & 0.60 \\
\hline \multicolumn{6}{c}{ Fuente: Elaboración propia } \\
\hline
\end{tabular}

\section{El modelo estructural}

El ajuste del modelo estructural fue evaluado inicialmente a través del error de aproximación cuadrático medio (RMSEA) el cual presenta un nivel de 0.077 y de acuerdo con Browne \& Cudeck (1992) si bien valores de 0.05 o menos indican un buen ajuste, valores de 0.08 o menores corresponden a un ajuste razonable del modelo. Posteriormente se calculó el índice de ajuste comparativo (CFI) del modelo, que en este caso experimentó un valor de 0.935 , con lo cual se establece que este modelo presenta un ajuste aceptable de acuerdo a este indicador (Medsker et al., 1994). Finalmente respecto a la bondad de ajuste, el modelo propuesto experimentó un nivel de 0.924 en el índice de Tucker-Lewis, el cual se encuentra por encima del nivel recomendable de 0.90 (Hair et al., 2010).

\section{Resultados del modelo}

Como se puede observar en la figura 2, las cargas factoriales se encuentran por arriba de 0.7 para cada uno de los constructos. La confianza en la plataforma resultó tener una relación positiva y significativa con la lealtad del clienta hacia la empresa $(\beta=0.32)$, con lo cual la hipótesis 2 no se rechaza. Además se muestra que la satisfacción del cliente es el constructo que más influencia ejerce en la lealtad a la plataforma $(\beta=0.46)$ con un nivel de significancia menor a 0.001 , con lo cual la hipótesis 3 fue validada. Sin embargo, la relación entre la usabilidad de la plataforma con la lealtad resultó no significativa, por lo cual la hipótesis 1 se rechazó. Adicionalmente se debe mencionar el resultado obtenido en el R2 ajustado del modelo estructural, el cual expresa que las variables latentes explican un $66 \%$ de la varianza de la variable dependiente lealtad del cliente.

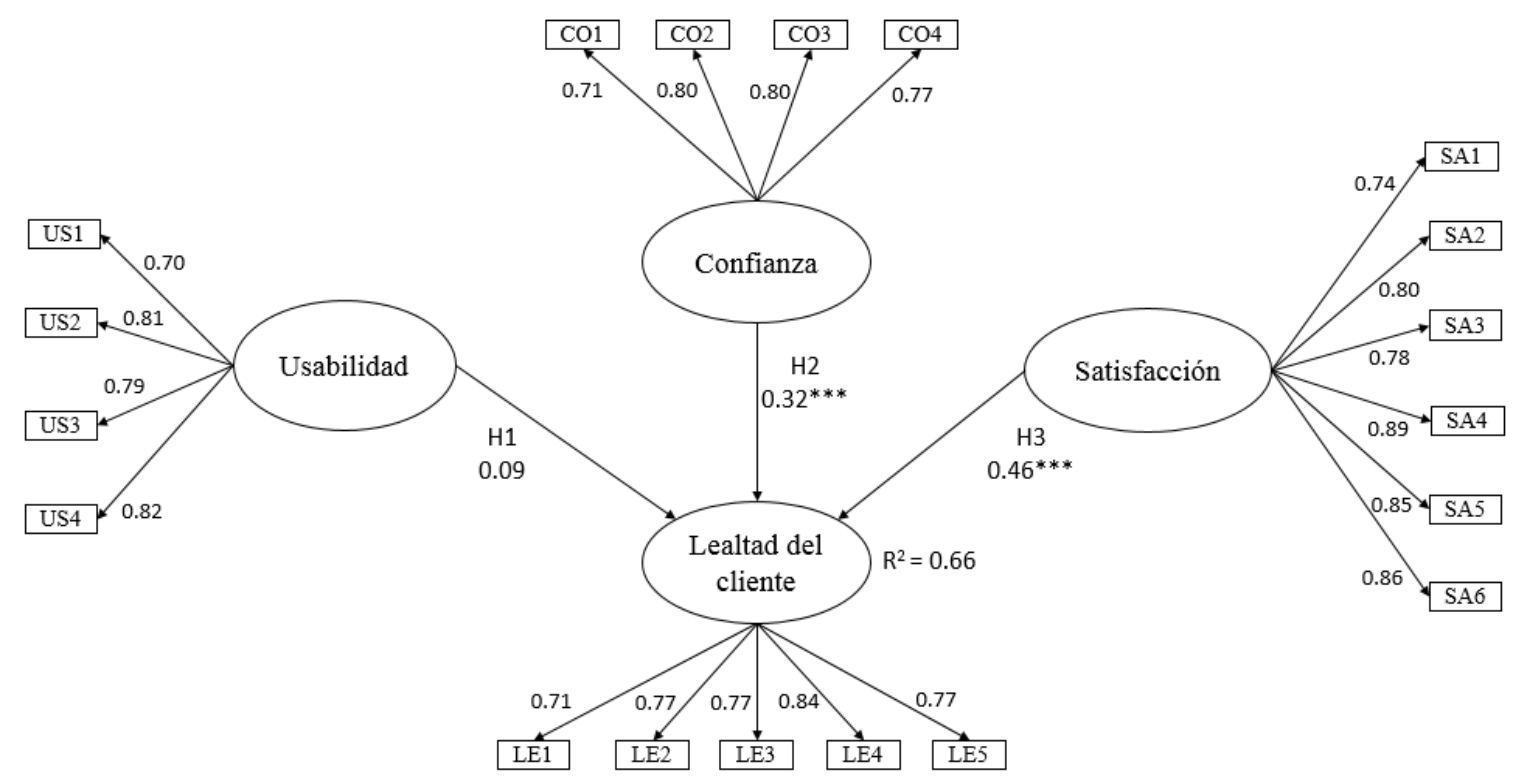

Figura 2. Modelo final propuesto para medir la lealtad del cliente.

*** Significativa al 0.001

Fuente: Elaboración propia

\section{DISCUSIÓN}


En esta investigación se relaciona a las variables independientes usabilidad, la confianza y la satisfacción de los clientes y su influencia en la lealtad hacia una tienda en línea en el contexto de la pandemia generada por el COVID-19. En la literatura revisada, se encontraron investigaciones que relacionan a la lealtad hacia una empresa con la satisfacción de los clientes (Aslam et al., 2020; López et al, 2018; Phani y Prasanna, 2016), con la calidad del servicio (Goutam y Gopalakrishna, 2018) y con la confianza en la empresa en línea (Othman et al., 2019), sin embargo no se encontraron investigaciones que relacionaran de manera conjunta a la lealtad en una tienda en línea con usabilidad, la confianza y la satisfacción en el contexto de países en desarrollo como es el caso de México. Entrelosresultadosdelainvestigación,lahipótesis 1 fuerechazadadebidoaquenoresultóestadísticamente significativa. Lo anterior recuerda al trabajo realizado por Celia e Isaza (2019), quienes no encontraron una relación evidente entra la usabilidad de la página electrónica con la imagen de marca de una empresa colombiana. Por otra parte se comprobó la hipótesis 2 , al verificarse una relación positiva y significativa entre la confianza en la tienda en línea con la lealtad a la empresa $(\beta=0.32)$. Este resultado se encuentra en sintonía con los hallazgos presentados por Molinillo, et al., (2016), quien reportó que el estado afectivo, la satisfacción y la confianza influyen en la lealtad de los clientes de una tienda minorista en línea, del sector de prendas de vestir en España. Así mismo este resultado se asemeja a lo obtenido por Piscoya (2019), quien concluyó que existe una correlación positiva y significativa entre las diferentes dimensiones de la confianza de los consumidores con la lealtad de una empresa bancaria en Perú. La hipótesis 3 no fue rechazada al verificarse la relación positiva y significativa entre las variables satisfacción y lealtad de los clientes. Los resultados del modelo muestran que ésta variable fue la que mayor impacto generó sobre la lealtad de los clientes $(\beta=0.46)$. Estos hallazgos coinciden con los reportados por Lacaci (2017), quién encontró mediante la tecnica del modelado de ecuaciones estructurales, una influencia de la satisfacción sobre la lealtad de los clientes, en una investigación empírica realizada entre 400 clientes de una empresa minorista con ventas en linea en España. Finalmente, los resultados de la presente investigación ayudan a cubrir lagunas detectadas en la literatura, relacionadas con verificar empiricamente la influencia de la confianza, la satisfacción y la usabilidad de las tiendas en linea con la lealtad de los clientes (López, et al., 2018; Molinillo, et al., 2016).

\section{CONCLUSIONES}

La presente investigación se propuso como objetivo verificar un modelo que relaciona la usabilidad, la confianza y la satisfacción con la lealtad de los clientes de una plataforma digital de compra venta de mercancías, a través del modelado de ecuaciones estructurales. Para lograr el objetivo se planteó un modelo de medida cuyos constructos cumplieron los requisitos de validez convergente y discriminante, mediante los indicadores de alfa de Cronbach, fiabilidad compuesta y varianza media extraída, así mismo se superó la prueba de Fornell y Larcker. Posteriormente, el modelo estructural resultó con un ajuste aceptable de acuerdo a los indicadores presentados, el RMSEA, CFI y el índice de Tucker-Lewis. De acuerdo los resultados obtenidos las hipótesis 2 y 3 encontraron respaldo mientras que la hipótesis 1 fue rechazada, debido a que la relación de la usabilidad de la plataforma con la lealtad del cliente no fue estadísticamente significativa. Estos resultados contribuyen a mostrar evidencia empírica de la influencia que tiene la confianza y la satisfacción que sobre una empresa de comercio digital tienen sus clientes para influir en la lealtad hacia ésta, en tiempos en que existen restricciones de movilidad de las personas para realizar sus compras de sus mercancías y servicios de manera física, debido a la pandemia generada por el COVID-19. Por lo cual, una de las implicaciones gerenciales que resultan de esta investigación, es que los gerentes de tiendas en línea deben procurar generar confianza de sus clientes en sus servicios digitales, así como realizar las acciones necesarias con sus clientes internos, invertir 
en sistemas de seguridad en su tienda en línea y ofrecer servicios los servicios post venta adecuados con el propósito de generar la satisfacción de sus clientes que fomentan la lealtad hacia sus empresas. Entre las limitaciones que cuenta esta investigación, se debe mencionar que la muestra estuvo conformada en su mayoría por personas jóvenes, el 83\% manifestó contar con 40 años o menos de edad. Así mismo, la muestra se obtuvo solamente de un estado de México, Por lo cual futuras investigaciones podrían tener como propósito verificar si esta relación entre los constructos, se verifica para personas con mayor edad y en otros estados de la República Mexicana.

\section{REFERENCIAS}

1. Anderson, R. y Srinivasan, S. (2003). E-Satisfaction and E-Loyalty: A Contingency Framework. Psychology and Marketing, 20 (2), 123-138. https://doi.org/10.1002/mar.10063

2. Aslam, W., Hussain, A., Farhat, K., y Arif, I. (2020). Underlying Factors Influencing Consumers' Trust and Loyalty in E-commerce. Business Perspectives and Research, 8 (2), 186-204. https:// doi.org/10.1177/2278533719887451

3. Asociación de Internet MX. (2019). Asociación de Internet MX | Estudios y Hábitos Digitales.

4. Badía, D., José, M., y Arizón, B. (2018). Calidad del e-servicio y confianza online: análisis de las plataformas Airbnb y Booking Quality of online service and online confidence: Airbnb and Booking. In zaguan.unizar.es. http://zaguan.unizar.esautor/es

5. Bauer, H., Grether, M., y Leach, M. (2002). Customer Relations Through the Internet. Journal of Relationship Marketing, 1(2), 39-55. https://doi.org/10.1300/J366v01n02_03

6. Browne, M. y Cudeck, R. (1992). Alternative Ways of Assessing Model Fit. In Sociological Methods \& Research (Vol. 21, Issue 2, pp. 230-258). https://doi.org/10.1177/0049124192021002005

7. Cascos, F. (2009). Estadística Descriptiva. http://www.est.uc3m.es/icascos/esp/resumen descriptiva.pdf

8. Celia, M., y Isaza, M. (2019). El E-commerce y el impacto de la Navegabilidad en la imagen de marca Caso de estudio Olimpica S.A. http://repository.cesa.edu.co/handle/10726/2208

9. Chou, S., Chen, C., y Lin, J. (2015). Female online shoppers: Examining the mediating roles of e-satisfaction and e-trust on e-loyalty development. Internet Research, 25(4), 542-561. https://doi. org/10.1108/IntR-01-2014-0006

10. Colmenares, O y Saavedra, J. (2007). Aproximación teórica de la lealtad de marca: enfoques y valoraciones. 7 (82), 69-81. http://www.ehu.eus/cuadernosdegestion/documentos/725.pdf

11. Cronbach, L. (1951) Coefficient alpha and the internal structure of tests. In psychometrika. 16 (3), https://doi.org/10.1007/BF02310555.

12. Cuevas, H. (noviembre, 2020). La pandemia dispara el interés por las plataformas digitales y marketplace un 50\%. https://www.hosteltur.com/comunidad/nota/024269_la-pandemia-disparael-interes-por-las-plataformas-digitales-y-marketplace-un-500.html

13. Cupani, M. (2012). Análisis de ecuaciones estructurales: Conceptos, etapas de desarrollo y un ejemplo de aplicación. Revista Tesis, 2, 186-199.

14. Fornell, C. y Larcker, D. (1981). Evaluating Structural Equation Models with Unobservable Variables and Measurement Error. Journal of Marketing Research, 18(1), 39-50.

15. Fuentes, M., Moliner, B, y Gil, I. (2017). El efecto del sector de actividad en el proceso de satisfacción con la tienda. XXIX Congreso de Marketing Aemark 2017, 1356-1371. https:// idus.us.es/bitstream/handle/11441/78055/el_efecto_del_sector_de_actividad_en_el_proceso_de_ satisfaccion.pdf? sequence $=1 \&$ isAllowed $=\mathrm{y}$

16. González, J. (2020). Comercio electrónico en China y México: surgimiento, evolución y perspectivas. México y la Cuenca del Pacífico, 9 (27), 53-84. https://doi.org/10.32870/mycp. v9i27.688

17. Goutam, D., y Gopalakrishna, B. (2018). Customer loyalty development in online shopping: An integration of e-service quality model and commitment-trust theory. Management Science Letters, 8(11), 1149-1158.

18. Hair, J., Black, W., Babin, B. y Anderson, R. E. (2010). Multivariante data analysis (5ta ed.). Prentice Hall. 
19. Hampton, W., y Koufaris, M. (2005). The effect of Web site perceptions on initial trust in the owner company. International Journal of Electronic Commerce, 10 (1), 55-81. https://doi.org/10.1080/1 0864415.2005.11043965 53

20. Hernández, R., Baptista, P. y Hernández, C. (2004). Metodología de la Investigación. McGrawHill Interamericana.

21. Hernández, R., Fernández, C. y Baptista, P. (2010). Metodología de la investigación. Quinta Edición. McGraw-Hill.

22. Instituto Nacional de Estadística y Geografía INEGI. (2015). Número de habitantes. Hidalgo. http://cuentame.inegi.org.mx/monografias/informacion/hgo/poblacion/

23. Instituto Nacional de Estadística y Geografía INEGI. (2018). Comercio Electrónico. Comercio Electrónico. https://www.inegi.org.mx/teMmas/vabcoel/

24. Kim, D., y Benbasat, I. (2010). Trust-Assuring Arguments in B2C E-commerce: Impact of Content, Source, and Price on Trust. Journal of Management Information Systems, 26(3), 175-206. https:// doi.org/10.2753/MIS0742-1222260306

25. Kim, D. (2009), Trust and satisfaction, two stepping stones for successful e-commerce relationships: A longitudinal exploration, Information Systems Research, 20 (2), 237-257

26. Kotler, P. y Armstrong, G. (2008). Principios de Marketing. Pearson Educación. Madrid.

27. Manzano, J., Pérez, R., Mafé, C., y Blas, S. (2010). Factores determinantes de la lealtad en el comercio electrónico b2c. Aplicación a la compra de billetes de avión. Revista Española de Investigación de Marketing ESIC, 14 (2), 113-142. https://aemark.org/esic/2010/100917_132147_E.pdf

28. Marín, M. (2017). Usabilidad en la compra online de mercadona: análisis y propuesta. [Tesis de grado]. https://idus.us.es/bitstream/handle/11441/63312/TFGDEFINITIVO. pdf? sequence $=1 \&$ isAllowed $=y$

29. Medsker, G., Williams, L. y Holahan, P. J. (1994). A review of current practices for evaluating causal models in organizational behavior and human resources management research. Journal of Management, 20(2), 439-464.

30. Müggenburg, M., y Pérez, I. (2007). Los maestros escriben Tipos de estudio en el enfoque de investigación cuantitativa. Revista Enfermería Universitaria ENEO-UNAM, 4 (1), 35-38. http:// www.redalyc.org/pdf/3587/358741821004.pdf

31. Organización para la Cooperación y el Desarrollo Económicos OCDE. (2018). Acerca de la OCDE - OECD. https://www.oecd.org/acerca/

32. Ortega, V. (2020). Efectos del Covid-19 en el comportamiento del consumidor: Caso Ecuador. Retos Revista de Ciencias de la Administración y Economía, 10 (20), pp. 233-247. https://doi. org/10.17163/ret.n20.2020.03

33. Othman, A. K., Faizah, L., Hassan, A., Anuar, M., Ibrahim, M., Saripin, M. S., Sazatul, N., Sapuan, A., Alam, S. y Roslan, Z. N. (2019). Factors that influence customer loyalty in using e-commerce. In Journal of Islamic Management Studies, 2 (2), 43-58.

34. Parra, L. (2019). Nivel de satisfacción de clientes en compras en tienda virtual, caso Telltex SA. https://repository.ean.edu.co/handle/10882/9956

35. Pérez, S. (2018). La usabilidad de las páginas web de ecommerce. Análisis y propuesta de mejora de www.elcorteingles.com. [Tesis de grado]. https://idus.us.es/bitstream/handle/11441/79740/ TFG\%20Salom \%c3\%a9\%20P\%c3\%a9rez $\% 20$ Pallar\%c3\%a9s.pdf?sequence=1\&isAllowed=y

36. Peter, P. y Olson, J. (2006). Comportamiento del consumidor y estrategia de marketing. 7 edic., McGraw-Hill Interamericana.

37. Phani, B. y Prasanna, K. (2016). Customer Loyalty on E-Commerce. International Journal of Management Research \& Review, 6 (4), 2249-7196. www.ijmrr.com

38. Piscoya, D. (2019). La Confianza y lealtad en el uso de los Servicios Bancarios Virtuales Del Banco De Crédito Sucursal Lambayeque. [Tesis de grado]. http://54.165.197.99/ bitstream/20.500.12423/2028/1/TL_PiscoyaRuizDiana.pdf

39. Plasencia, X. (2018). La predicción de la lealtad online de los jóvenes en el Turismo. [Tesis de grado]. https://riull.ull.es/xmlui/bitstream/handle/915/12336/La+prediccion+de+la+lealtad+onlin e+de+los+jovenes+en+el+Turismo.pdf? sequence $=1$

40. Ramirez, D. (2015). Estudio del comportamiento del consumidor de comercio electrónico y su grado de satisfacción en las compras online. UTMACH, Unidad Académica de Ciencias Empresariales, Machala, Ecuador. http://186.3.32.121/handle/48000/3829 
41. Rodríguez, I., De Haro, G. y Somalo, I. (2017) Estudio de la usabilidad web en marcas de moda españolas mediante la herramienta de análisis heurístico Sirius. https://dialnet.unirioja.es/descarga/ articulo/6432259.pdf

42. Sánchez, J. y Montoya, L. (2016). Factores que afectan la confianza de los consumidores por las compras a través de medios electrónicos. Pensamiento y gestión. 40. https://doi.org/10.14482/ pege. 40.8809

43. Sánchez, W. (2011). La usabilidad en Ingeniería de Software: definición y características. Ing-Novación. Reporte de Investigación, 2, 7-21. http://www.redicces.org.sv/jspui/ bitstream/10972/1937/1/2

44. Sanz, S., Ruiz, C. y Pérez, I. (2009). Conceptos, dimensiones y antecedentes de la confianza en los entornos virtuales. Teoría y Praxis, 5 (6), 31-58. https://doi.org/10.22403/uqroomx/typ06/02

45. Shopify (2015). Comportamiento del consumidor y lealtad a la marca. https://www.shopify.com. $\mathrm{mx} / \mathrm{blog} / 43876037$-la-importancia-de-la-lealtad-de-marca-en-el-posicionamiento-de-un-negocio

46. Thompson, I. (2006). La Satisfacción del Cliente. Red Med, 6. https://www.procase.cl/Demos/ tmk/docs/Satisfacci\%C3\%B3n\%20al\%20Cliente.pdf

47. Ubaque, J. (2020). Análisis del comportamiento del consumidor frente a los efectos de la pandemia del COVID19 en artículos de la canasta básica en las cadenas de grandes superficies de Colombia. Universidad militar nueva granda. https://repository. unimilitar.edu.co/bitstream/handle/10654/36332/UbaqueGutierrezJavierGuillermo2020. pdf? sequence $=3 \&$ is Allowed $=y$

48. Vega, J. (2015). Determinantes de la confianza del consumidor hacia el comercio electrónico: Una aplicación al caso de Puerto Rico. Esic Market Economics and Business Journal, 46, 149-172. https://doi.org/10.7200/esicm.150.0461.3e

49. Wang, Y. y Emurian, H. (2005). An overview of online trust: Concepts, elements, and implications. Computers in Human Behavior 21 (1), 105-125. https://doi.org/10.1016/j.chb.2003.11.008 